\title{
O COMÉRCIO DA LÓGICA
}

Frank Thomas Sautter

UFSM

Resumo: Conclusão e Premissas negociam peças de informação entre si. Conclusão carece delas, Premissas dispõe delas. $\mathrm{O}$ saldo entre carência e disponibilidade exige aferição. O método heterogêneo de prova para a Lógica Sentencial Clássica, aqui desenvolvido, implementa esta metáfora. Ele pode ser empregado solitário, cooperativa, ou competitivamente.

Palavras-Chave: Informação; informação errada; kanban; soroban.

Abstract: Conclusion and Premises trade pieces of information among themselves. Conclusion lacks them, Premises supplies them. The balance between lack and availability requires admeasurement. The heterogeneous proof method for Classical Sentential Logic developed here implements this metaphor. It can be employed solitarily, cooperatively, or competitively.

Keywords: Information; misinformation; kanban; soroban.

Wo Geld isch, isch au drDeif; wokoinsisch, do ischersogarzwoi mol. Onde há dinheiro, ali também está o Diabo; onde não há, ali ele está inclusive duas vezes.

(Provérbio sábio)

\section{Introdução}

Conclusão e Premissas são agentes no comércio da Lógica; informação é o bem que negociam entre si. Conclusão carece de informações, e sinaliza isso para Premissas. Premissas dispõem de informações, e sinaliza isso para Conclusão. Essa dupla sinalização é o kanban ${ }^{1}$ da Lógica. O saldo entre carência e disponibilidade geralmente não é evidente, e exige aferição. Essa aferição é o soroban ${ }^{2}$ da Lógica.

1 'Kanban' é um termo técnico utilizado em administração da produção para indicar um sistema de sinalização, à base de cartões, empregado no fluxo da produção.

${ }^{2}$ 'Soroban' é o ábaco japonês. 
O método heterogêneo de prova para a Lógica Sentencial Clássica (LSC), aqui desenvolvido, utiliza a forma normal conjuntiva para implementar esta metáfora. $\mathrm{Na}$ primeira seção apresento as bases teóricas do método; na segunda seção, sua implementação para o teste de validade de argumentos da LSC. As mesmas figuras e as mesmas regras de manipulação dessas figurações empregadas no teste de validade dual, ou seja, validade e validade dual são distintas faces da mesma moeda. $\mathrm{Na}$ terceira seção apresento, em linhas gerais, a correção e a completude forte do método. Na quarta seção apresento o fenômeno da dualidade; na quinta seção, a aplicação do método para o teste de validade dual de argumentos da LSC. Na sexta seção examino diferentes modos de aplicação do método: seja como um método solitário, seja como um método cooperativo, seja como um método competitivo. $\mathrm{Na}$ sétima e última seção esboço uma medida para a complexidade de argumentos baseada no método e, com ela, uma hierarquia da complexidade de argumentos.

O leitor familiarizado com os métodos heterogêneos de prova reconhecerá, neste trabalho, minha dívida em relação aos aportes lógicos de Charles Sanders Peirce.

\section{Informação e forma normal conjuntiva}

A verdade admite, no seu uso coloquial, comparação e gradação; por exemplo, qualificamos afirmações como 'meias verdades' e dizemos, de algumas, que são mais verdadeiras do que outras. No seu uso técnico clássico uma 'meia verdade' e uma 'manifesta falsidade' não diferem substancialmente, tampouco há lugar para afirmações mais verdadeiras do que outras; no seu uso técnico clássico a verdade não é apta a comparações ou gradações ${ }^{3}$. Haver um modo de comparar e graduar verdades em seu uso técnico facilitaria o processo de aperfeiçoamento de argumentos, mediante o fortalecimento ou o enfraquecimento de afirmações ${ }^{4}$.

A informação também admite, no seu uso coloquial, comparação e gradação; por exemplo, qualificamos afirmações de 'pouco informativas' e dizemos, de algumas, que são mais informativas do que outras. Porém, ao contrário da verdade, há um uso técnico clássico da informação naturalmente apto a comparações e gradações. Isso decorre das seguintes considerações de caráter lógico ${ }^{5}$ :

\footnotetext{
3 Há sistemas lógicos não clássicos que admitem comparação e gradação da verdade.

${ }^{4}$ AUTOR e SANZ (2013) discutem o aperfeiçoamento de argumentos inválidos da LSC, e AUTOR e MENDONÇA (2014) discutem o aperfeiçoamento de argumentos válidos da LSC. Para uma visão geral sobre a possibilidade de aperfeiçoamento de argumentos, sua importância, e sua realização no interior da lógica formal consultar AUTOR (2012).

${ }^{5}$ CORCORAN (1998) faz uma revisão de abordagens informacionais à lógica formal.
} 
- A informação veiculada por uma conjunção é a soma mereológica das informações veiculadas pelos seus conjuntivos.

- Toda sentença da LSC é logicamente equivalente a uma sentença em forma normal conjuntiva, ou seja, a uma conjunção cujos conjuntivos são disjunções de literais (sentenças atômicas ou negações de sentenças atômicas) ${ }^{6}$.

Acrescente-se a isso a seguinte caracterização informacional de argumento válido: Um argumento é válido se e somente se cada informação veiculada pela conclusão também é veiculada por ao menos uma premissa.

Uma possível objeção levantada contra o emprego da noção de informação decorre da exigência de haver átomos absolutos de informação. Tal exigência não pode ser satisfeita, pois a simplicidade informacional é distinta da simplicidade lógica ${ }^{7}$. Felizmente, átomos absolutos de informação não são necessários para os propósitos da Lógica. É suficiente lidar com informação relativa.

Essa informação relativa, aplicada a um argumento, traduz-se em apenas considerar as sentenças atômicas desse argumento. O que se faz é obter, com respeito a cada sentença do argumento, a forma normal conjuntiva completa com respeito às sentenças atômicas que ocorrem nele. $\mathrm{O}$ seguinte exemplo ajudará a entender o ponto. Seja a instância de modus ponens cujas premissas são ( $\mathrm{p} \supset \mathrm{q})$ e p, e cuja conclusão é q, para p e q sentenças atômicas. O correspondente desse argumento, em forma normal conjuntiva, é constituído pela premissa ( $\neg \mathrm{p} \vee \mathrm{q}$ ), pela premissa $\mathrm{p}$, e pela conclusão $\mathrm{q}$, todas essas sentenças correspondendo a formas normais conjuntivas degeneradas. Até o momento não é possível comparar as sentenças quanto às informações que veiculam, pois elas falam de coisas distintas: uma delas fala de $\mathrm{p}$ e de $\mathrm{q}$, outra fala somente de p, e outra fala somente de q. Colocamo-las em forma normal conjuntiva completa com respeito às sentenças atômicas que ocorrem no argumento, a saber, p e q. A primeira premissa permanece inalterada, a saber, $(\neg \mathrm{p} \vee \mathrm{q})$; a segunda premissa recebe a forma logicamente equivalente $((p \vee q) \wedge(p \vee \neg q))$; e a conclusão recebe a forma logicamente equivalente $((p \vee q) \wedge(\neg p \vee q))$. O argumento é válido, porque as informações veiculadas pela conclusão, a saber, as informações veiculadas por ( $p \vee q)$ e $(\neg p \vee q)$, também são veiculadas pelas premissas, uma por uma premissa, a outra pela

${ }^{6}$ Há casos degenerados de forma normal conjuntiva: há casos em que há um único conjuntivo, por exemplo, $(p \vee \neg p)$, e há casos em que em um conjuntivo há um único disjuntivo, por exemplo, $q \wedge(p$ $\vee \neg p)$.

7 Se $\alpha$ é uma sentença atômica, ela é, em geral, mais informativa do que ( $\alpha \vee \beta)$. Portanto, se escolhermos adequadamente as sentenças, sempre se pode, por disjunção, obter sentenças menos informativas do que qualquer uma das sentenças até o momento consideradas. 
outra premissa; além disso, há uma informação irrelevante na segunda premissa, ou seja, o argumento pode ser melhorado.

O procedimento acima ilustrado pode, apesar de utilizar símbolos e não figuras, ser considerado um método heterogêneo de prova, porque, para os fins do método, os conjuntivos das formas normais conjuntivas completas das sentenças do argumento são manipulados como se fossem figuras: as operações de reconhecimento de conjuntivos e de comparação dos mesmos não exigem nada além daquilo que exigimos de operações com figuras ${ }^{8}$.

A seguir, mostrarei a implementação dessa técnica mediante a representação por figuras e regras para a manipulação das mesmas. O método pode ser empregado como um jogo, independente do seu vínculo com a Lógica; ele pode ser utilizado para educar no uso de regras. Seja como método heterogêneo de prova, seja como puro jogo, ele é apto a ser praticado solitário, cooperativa, ou competitivamente.

\section{O kanbane o soroban da Lógica - teste de validade}

Apresentarei, primeiro, os elementos gráficos do método e o que eles representam; a seguir, apresentarei as regras de manipulação desses elementos gráficos e como elas operam para a determinação da validade ou invalidade de argumentos da LSC. Também apresentarei dois exemplos de aplicação do método, um deles envolvendo um argumento válido, o outro envolvendo um argumento inválido.

Os seguintes elementos gráficos são utilizados:

1) Barra de inferência: barra com o sinal '+' na sua extrema esquerda. Acima dela representamos a(s) premissa(s); abaixo dela, a conclusão. Na Figura 2(a) há uma barra de inferência, acima dela a(s) premissa(s) representada(s) por três quadrados, dois deles formando um bloco e o outro formando um segundo bloco, e abaixo dela a conclusão representada por um único quadrado e, portanto, um único bloco. $\mathrm{Na}$ Seção 4 utilizarei uma barra dual, uma barra com o sinal '-' na sua extrema esquerda.

2) Quadrados: representam literais. No canto inferior esquerdo indicamos a sentença atômica à qual o literal está vinculado ${ }^{9}$ e no canto

\footnotetext{
${ }^{8}$ AUTOR (2013) aplicou essas ideias ao desenvolvimento de um método heterogêneo de prova para a LSC; o método aqui apresentado é um desenvolvimento natural daquele método. AUTOR (2015) aplicou as mesmas ideias ao desenvolvimento de um método heterogêneo de prova para a Lógica de Predicados Monádicos de Primeira Ordem; nesse caso foi necessário recorrer à forma normal conjuntiva e à forma normal de Herbrand.

${ }^{9}$ Essa indicação é dada por um numeral. Suponha que as sentenças atômicas estão dadas por uma enumeração e que a indicação corresponde à posição de cada uma delas na enumeração. Para facilitar a compreensão, seja 'pi' a sentença atômica cuja indicação é dada pelo numeral 'i'.
} 
superior direito indicamos a polaridade do literal, de tal modo que o sinal ' + ' indica a própria sentença atômica à qual o literal está vinculado e o sinal '-' indica a negação da sentença atômica à qual o literal está vinculado ${ }^{10} . \mathrm{Na}$ Figura 2(a) o primeiro bloco de premissa(s) é constituído por $\neg \mathrm{p}_{1}$ seguido de p2.

3) Contiguidade de quadrados: representa a disjunção inclusiva dos literais representados pelos quadrados contíguos. Na Figura 2(a) o primeiro bloco de premissa(s) representa $\left(\neg \mathrm{p}_{1} \vee \mathrm{p}_{2}\right)$.

4) Separação entre blocos: representa a conjunção das sentenças relacionadas aos blocos separados. Na Figura 2(a) a separação entre o primeiro bloco e o segundo bloco de premissa(s) representa $\left(\left(\neg \mathrm{p}_{1} \vee \mathrm{p}_{2}\right) \wedge \mathrm{p}_{1}\right)$.

Os únicos elementos gráficos do método estão dados acima, portanto o método opera com a negação, a conjunção, e a disjunção inclusiva como conetivos primitivos. E não apenas isso: o método requer que as sentenças sejam apresentadas em forma normal conjuntiva ${ }^{11}$. Portanto, há um préprocessamento no qual sentenças são transformadas em sentenças logicamente equivalentes que empregam apenas a negação, a conjunção, e a disjunção inclusiva, caso empreguem outros conetivos. Além disso, um segundo préprocessamento as transforma em sentenças logicamente equivalentes que estão em forma normal conjuntiva. Confio ao leitor a realização de tais préprocessamentos.

O método opera com dois conjuntos de regras: as regras de aferição de informação e a regra de comércio de informação. Elas correspondem aproximadamente às regras operacionais e às regras estruturais, respectivamente, do cálculo de sequentes.

A regra de comércio de informação permite o apagamento de um bloco que ocorra acima da barra de inferência (na(s) premissa(s) e, portanto, disponível para uso) e que também ocorra abaixo da barra de inferência (na conclusão e, portanto, cujo uso é requerido).

As cinco regras de aferição de informação estão dadas na Figura 1. Elas permitirão uma melhor comparação entre a informação disponível na(s) premissa(s) (acima da barra de inferência) e a informação requerida na conclusão (abaixo da barra de inferência). Elas permitem uma melhor aferição do saldo nesse comércio entre Premissas e Conclusão.

\footnotetext{
${ }^{10} p_{i}^{\prime}$ é o literal representado pelas indicações 'i' e 't', ' $\neg p_{i}^{\prime}$ é o literal representado pelas indicações 'i' e ':

11 Não é compulsória a apresentação em forma normal conjuntiva completa em relação às sentenças atômicas do argumento, pois as regras de manipulação permitirão que essa forma normal conjuntiva completa seja obtida quando necessário.
} 
EXPANSĀO:

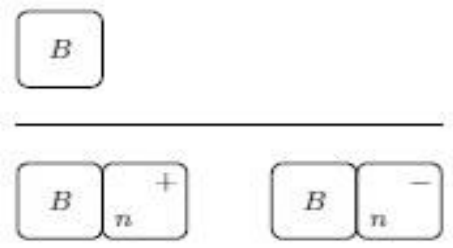

RETRAC̣ĀO:

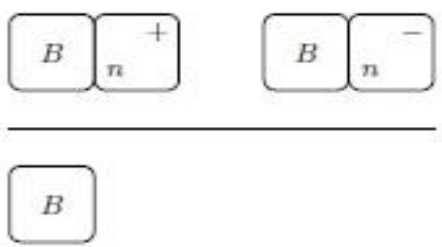

\section{PERMUTAC̄ĀO:}

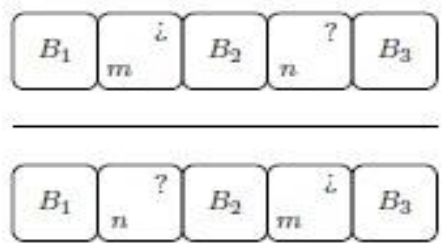

CRIAC̄ĀO:

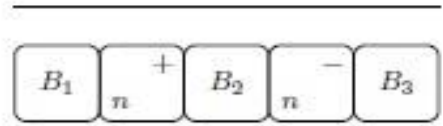

EXTINC̄ĀO:

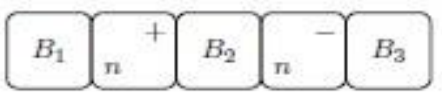

Figura 1: Regras de aferição de informação. 
A regra de EXPANSÃO permite a substituição, no setor da(s) premissa(s) ou no setor da conclusão, de um bloco B por dois blocos: um deles representando a disjunção do representado por $\mathrm{B}$ com $\mathrm{p}_{\mathrm{n}}$ e o outro a disjunção do representado por $\mathrm{B}$ com $\neg \mathrm{p}_{\mathrm{n}}$. Essa regra está sujeita à seguinte restrição: o bloco $B$ não pode ser vazio. Observe que se $p_{n}$ ou $\neg p_{n}$ já ocorre representado no bloco $\mathrm{B}$, a aplicação da regra é inócua.

A regra de RETRAÇÃO é o inverso da regra de EXPANSÃO, e, portanto, também requer que o bloco B não seja vazio.

$\mathrm{Na}$ regra de PERMUTAÇÃO os blocos $\mathrm{B}_{1}, \mathrm{~B}_{2}$, e $\mathrm{B}_{3}$ podem ser vazios, indicando que a permutação de quadrados num bloco pode ser legitimamente realizada independente da posição desses quadrados. O ponto de interrogação e o ponto de interrogação invertido indicam que a polaridade de um quadrado não interfere na sua aptidão para ser permutado.

$\mathrm{Na}$ regra de CRIAÇÃO os blocos $\mathrm{B}_{1}, \mathrm{~B}_{2}$, e $\mathrm{B}_{3}$ também podem ser vazios. Convencionou-se uma ordem para os demais quadrados (primeiro o positivo, depois o negativo) que pode ser facilmente alterada mediante aplicação da regra de PERMUTAÇÃO. A aplicação desta regra é especialmente útil quando se quer testar a tautologicidade de uma sentença; esse teste é efetuado examinando a validade de um argumento sem premissas e cuja conclusão é a própria sentença testada.

A regra de EXTINÇÃ̃O é o inverso da regra de CRIAÇÃO. Portanto, os blocos $\mathrm{B}_{1}, \mathrm{~B}_{2}$, e $\mathrm{B}_{3}$ também podem ser vazios, e a ordem dos demais quadrados também não é problema, uma vez que dispomos da regra de PERMUTAÇÃO.

Considere, a título de ilustração, os passos de prova da validade de instância de modus ponens cujas premissas, em forma normal conjuntiva, são $\left(\neg \mathrm{p}_{1} \vee \mathrm{p}_{2}\right)$ e $\mathrm{p}_{1}$ (acima da barra de inferência na Figura 2(a)), e cuja conclusão, em forma normal conjuntiva, é p 2 (abaixo da barra de inferência na Figura $2(a))^{12}$. Aplica-se a regra de EXPANSÃO à Figura 2(a) e se obtém a Figura 2(b). Aplica-se duas vezes a regra de PERMUTAÇÃO à Figura 2(b) e se obtém a Figura 2(c). Aplica-se a regra de comércio de informação à Figura 2(c) e se obtém a Figura 2(d). Aplica-se a regra de EXPANSÃO à Figura 2(d) e se obtém a Figura 2(e). Finalmente, aplica-se a regra de comércio de informação à Figura 2(e) e se obtém a Figura 2(f). O argumento é válido, porque Premissas disponibilizaram todas as informações requeridas por Conclusão.

Considere, agora, os passos de prova da invalidade de instância da falácia da negação do antecedente. A Figura 3(a) apresenta suas premissas acima da barra de inferência e sua conclusão abaixo da barra de inferência. $\mathrm{Na}$ passagem da Figura 3(a) até 3(f) utilizou-se, respectivamente, a regra de

${ }_{12}^{12}$ Para todos os propósitos não importa se os dois blocos acima da barra de inferência representam duas premissas ou uma única premissa. 
EXPANSÃO, duas vezes a regra de PERMUTAÇÃO, a regra de EXPANSÃO, e a regra de comércio de informação ${ }^{13}$. O argumento é inválido, porque uma informação requerida por Conclusão não está disponível pelas Premissas.

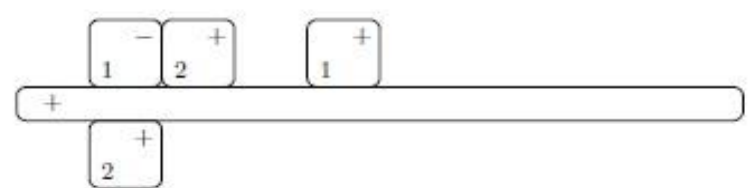

(a)

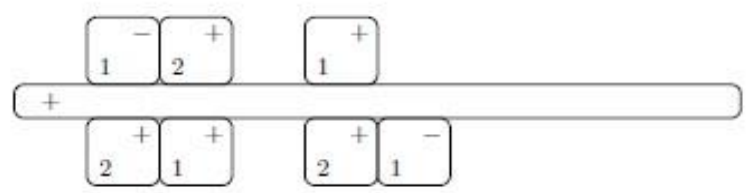

(b)

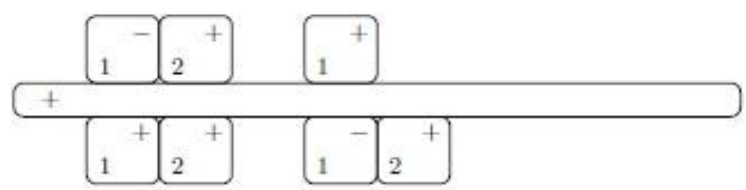

(c)

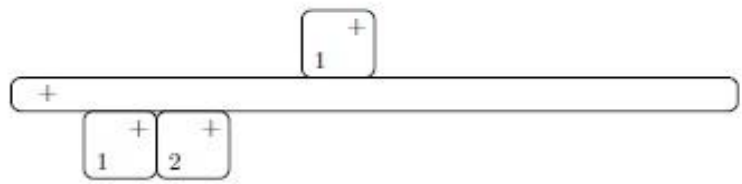

(d)

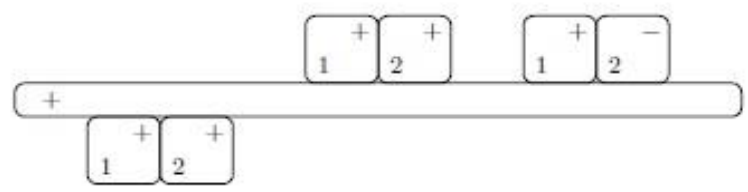

(e)

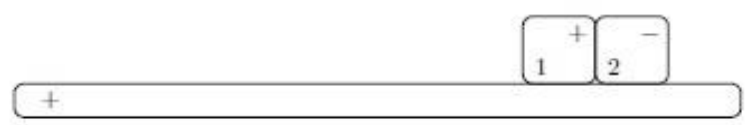

(f)

Figura 2: Passos de prova da validade de instância de modus ponens.

${ }^{13}$ Observe que houve a eliminação de dois blocos idênticos nas Premissas, o que não é previsto pelas regras de manipulação. Porém, isso não interfere no resultado, pois, caso o argumento fosse válido essa duplicidade poderia ser eliminada mediante a eliminação de ambas pela aplicação da regra de comércio de informação. 


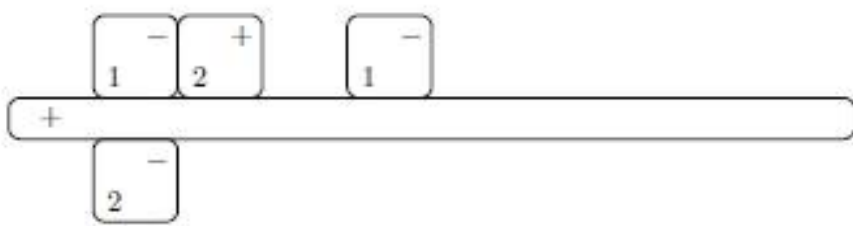

(a)

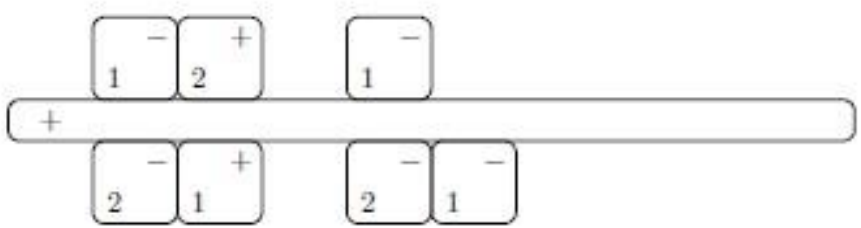

(b)

(c)

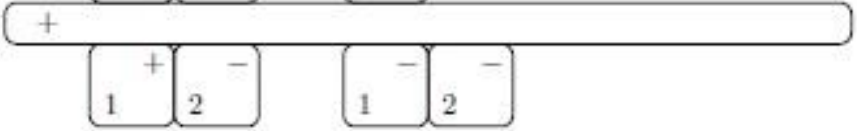

(d)

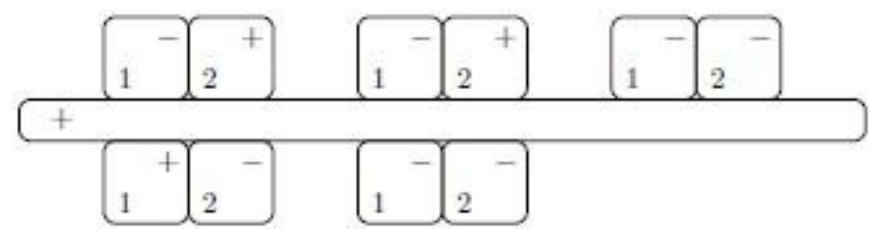

(e)

Figura 3: Passos de prova da invalidade de instância da falácia da negação do antecedente.

\section{Correção e completude forte}

A correção do método segue-se das seguintes considerações:

A correção da regra de EXPANSÃO segue-se em razão de que $(\alpha \wedge \beta)$, onde $\beta$ é uma tautologia, é acarretada por $\alpha$ (no presente caso ( $\alpha \wedge$ $\left.\left(\mathrm{p}_{\mathrm{n}} \vee \neg \mathrm{p}_{\mathrm{n}}\right)\right)$ é acarretada por $\left.\alpha\right)$ e $\left(\left(\alpha \wedge \beta_{1}\right) \vee\left(\alpha \wedge \beta_{2}\right)\right)$ é acarretada por $(\alpha \wedge$ 
$\left.\left(\beta_{1} \vee \beta_{2}\right)\right)$ (no presente $\operatorname{caso}\left(\left(\alpha \wedge p_{n}\right) \vee\left(\alpha \wedge \neg p_{n}\right)\right)$ é acarretada por $(\alpha \wedge$ $\left.\left.\left(\mathrm{p}_{\mathrm{n}} \vee \neg \mathrm{p}_{\mathrm{n}}\right)\right)\right)$. Uma contradição somente pode ser acarretada por um conjunto inconsistente de sentenças; a restrição à regra de EXPANSÃO visa evitar que uma contradição seja obtida exnibilo (no presente caso evita-se que $\left(\mathrm{p}_{\mathrm{n}} \vee \neg \mathrm{p}_{\mathrm{n}}\right)$ seja obtida exnibilo).

A correção da regra de RETRAÇÃO segue-se em razão de que os acarretamentos aludidos na correção da regra de EXPANSÃO são mútuos, ou seja, $\alpha$ é acarretada por $(\alpha \wedge \beta)$ e $\left(\alpha \wedge\left(\beta_{1} \vee \beta_{2}\right)\right)$ é acarretada por $\left(\left(\alpha \wedge \beta_{1}\right) \vee\right.$ $\left.\left(\alpha \wedge \beta_{2}\right)\right)$. A restrição à regra de RETRAÇÃO evita que ela atue, pelo menos parcialmente, como regra de comércio de informação.

A correção da regra de PERMUTAÇÃO segue-se em razão da simetria da disjunção.

A correção da regra de CRIAÇÃO segue-se da tautologicidade de $\left(\alpha \vee p_{n} \vee \beta \vee \neg p_{n} \vee \chi\right)$ e do acarretamento exnibilo de uma tautologia.

A correção da regra de EXTINÇÃO também se segue da tautologicidade de $\left(\alpha \vee p_{n} \vee \beta \vee \neg p_{n} \vee \chi\right)$ e de que a supressão de um conjuntivo tautológico não afeta a situação.

Para a prova da completude forte utilizarei o sistema axiomático fortemente completo para a LSC proposto por Bertrand Russell e Alfred N. Whitehead e simplificado por Paul Bernays (KNEALE; KNEALE, 1991, p. 532). Esse sistema utiliza a regra de substituição uniforme, a regra modus ponens (cuja validade pelo método aqui apresentado está dada na Figura 2 para uma instância), e dos seguintes axiomas, onde "p", "q", e "r" são sentenças atômicas:

$$
\begin{array}{ll}
\text { 1. } & ((\mathrm{p} \vee \mathrm{p}) \supset \mathrm{p}) \\
\text { 2. } & (\mathrm{q} \supset(\mathrm{p} \vee \mathrm{q})) \\
\text { 3. } & ((\mathrm{p} \vee \mathrm{q}) \supset(\mathrm{q} \vee \mathrm{p})) \\
\text { 4. } & ((\mathrm{q} \supset \mathrm{r}) \supset((\mathrm{p} \vee \mathrm{q}) \supset(\mathrm{p} \vee \mathrm{r})))
\end{array}
$$

É suficiente mostrar que tais axiomas são obteníveis pelo método aqui apresentado. Isso pode ser facilmente verificado, desde que se apresentem as formas normais conjuntivas completas de tais axiomas. Essas formas normais são as seguintes:

$$
\begin{aligned}
\text { 1. } & (\neg \mathrm{p} \vee \mathrm{p}) \\
\text { 2. } & (\neg \mathrm{q} \vee \mathrm{p} \vee \mathrm{q}) \\
\text { 3. } & (\neg \mathrm{p} \vee \mathrm{q} \vee \mathrm{p}) \wedge(\neg \mathrm{q} \vee \mathrm{q} \vee \mathrm{p}) \\
\text { 4. } & (\mathrm{q} \vee \neg \mathrm{p} \vee \mathrm{p} \vee \mathrm{r}) \wedge(\mathrm{q} \vee \neg \mathrm{q} \vee \mathrm{p} \vee \mathrm{r}) \wedge(\neg \mathrm{r} \vee \neg \mathrm{p} \vee \mathrm{p} \vee \mathrm{r}) \wedge(\neg \mathrm{r}
\end{aligned}
$$




\section{Dualidade e alternativa de informação errada}

As mesmas figuras e as mesmas regras de manipulação de figuras que foram utilizadas, na segunda seção, para o teste de validade de argumentos podem ser utilizadas para o teste daquela propriedade de argumentos que, abaixo, denominarei 'validade dual'.

O fenômeno da dualidade relaciona operações lógicas aos pares e, derivativamente, sentenças entre si. Para os propósitos do presente trabalho, considerarei as seguintes operações lógicas aos pares, duais uma da outra: a negação é a sua própria dual, e a conjunção e a disjunção inclusiva são duais uma da outra. A sentença dual $\mathrm{d}(\alpha)$ de uma dada sentença $\alpha$ é obtida mediante aplicação da seguinte função recursiva d:

- $\mathrm{d}(\alpha)$ é $\alpha$, se $\alpha$ é uma sentença atômica.

- $\mathrm{d}(\neg \alpha)$ é $\neg \mathrm{d}(\alpha)$.

- $\mathrm{d}(\alpha \wedge \beta)$ é $\mathrm{d}(\alpha) \vee \mathrm{d}(\beta)$.

- $\mathrm{d}(\alpha \vee \beta)$ é $\mathrm{d}(\alpha) \wedge \mathrm{d}(\beta)$.

O fenômeno da dualidade também relaciona as propriedades da validade e invalidade a duas outras propriedades de argumentos, a saber, a validade dual e a invalidade dual, respectivamente. Inicialmente caracterizarei essas duas últimas noções, assim como as duas noções tradicionais de validade e invalidade, em termos de valores de verdade:

- Um argumento é válido se, e somente se, necessariamente se as premissas são verdadeiras, a conclusão também é verdadeira.

- Um argumento é inválido se, e somente se, possivelmente as premissas são verdadeiras e a conclusão é falsa, ou seja, um argumento é inválido se, e somente se, ele não é válido.

- Um argumento é válido dual se, e somente se, necessariamente se as premissas são falsas, a conclusão também é falsa.

- Um argumento é inválido dual se, e somente se, possivelmente as premissas são falsas e a conclusão é verdadeira, ou seja, um argumento é inválido dual se, e somente se, ele não é válido dual.

Inválido e válido dual não são equivalentes, pois o argumento cuja única premissa é uma sentença e cuja conclusão é a dupla negação dessa sentença é válido e válido dual. A Figura 4 apresenta um diagrama de Venn dessas propriedades de argumentos, e todas as regiões desse diagrama são não vazias. 


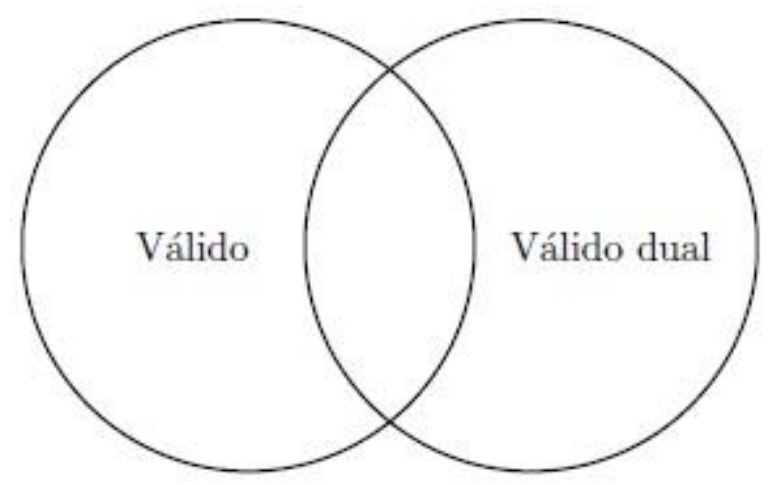

Figura 4: Diagrama de Venn de possíveis propriedades de um argumento.

Sanz (2008) demonstrou o seguinte resultado:

Teorema 1: Um argumento é válido se, e somente se, o seu argumento dual ${ }^{14}$ é válido dual.

Para os propósitos do presente trabalho, validade dual e invalidade dual, assim como validade e invalidade, podem receber a seguinte caracterização em termos informacionais:

- Um argumento é válido se, e somente se, toda informação requerida pela conclusão está disponibilizada em uma ou mais premissas.

- Um argumento é inválido se, e somente se, há uma informação requerida pela conclusão, mas que não está disponibilizada em nenhuma premissa.

- Um argumento é válido dual se, e somente se, toda alternativa de informação errada ${ }^{15}$ requerida pela conclusão está disponibilizada, como alternativa de informação errada, em uma ou mais premissas.

- Um argumento é inválido dual se, e somente se, há uma alternativa de informação errada requerida pela conclusão, mas que não está disponibilizada, como alternativa de informação errada, em nenhuma premissa.

${ }^{14} \mathrm{O}$ argumento dual de um dado argumento é aquele cuja conclusão é a dual da conclusão do argumento inicial e cujas premissas são as duais das premissas do argumento inicial.

${ }^{15}$ Explicarei, a seguir, o que se entende por 'alternativa de informação errada'. Ao utilizar a noção de informação errada (misinformation), rejeito a noção de informação fortemente semântica de Luciano Floridi (2004), a qual incorpora a tese da veracidade, ou seja, de que informação é informação verdadeira. É preciso distinguir misinformation de disinformation; esta, mas não aquela, incorpora um componente de intencionalidade. 
Foi visto, na seção anterior, o que se entende por 'informação' no contexto do presente método. O que se entende por 'alternativa de informação errada' será esclarecido mediante um exemplo.

O seguinte argumento, com as sentenças já expressas em termos de forma normal conjuntiva completa com respeito às sentenças atômicas do argumento, é uma instância de modus ponens, e, portanto, um argumento válido:

$$
\begin{gathered}
(\neg p \vee q) \\
(p \vee q) \wedge(p \vee \neg q) \\
\therefore(p \vee q) \wedge(\neg p \vee q)
\end{gathered}
$$

Os conjuntivos são informações e mediante uma mera inspeção visual percebe-se que toda informação requerida pela conclusão está disponibilizada em uma ou mais premissas: a informação veiculada por $(\mathrm{p} \vee \mathrm{q})$ está disponibilizada na segunda premissa e a informação veiculada por $(\neg \mathrm{p} \vee$ q) está disponibilizada pela primeira premissa (em rigor, coincide com a informação disponibilizada pela primeira premissa). O argumento é válido segundo a caracterização informacional de argumento válido aqui fornecida.

Considere o argumento dual:

$$
\begin{gathered}
(\neg p \wedge q) \\
(p \wedge q) \vee(p \wedge \neg q) \\
\therefore(p \wedge q) \vee(\neg p \wedge q)
\end{gathered}
$$

Cada disjuntivo é o que se entende por alternativa de informação errada. Mediante uma mera inspeção visual percebe-se que toda alternativa de informação errada requerida pela conclusão está disponibilizada, como alternativa de informação errada, em uma ou mais premissas: a alternativa de informação errada $(\mathrm{p} \wedge \mathrm{q})$ está disponibilizada na segunda premissa e a alternativa de informação errada $(\neg \mathrm{p} \wedge \mathrm{q})$ está disponibilizada pela primeira premissa (em rigor, coincide com a alternativa de informação errada disponibilizada pela primeira premissa). O argumento é válido dual segundo a caracterização informacional de argumento válido dual aqui fornecida.

Na próxima seção verei como essas ideias se materializam no método heterogêneo de prova aqui proposto. 


\section{O kanbane o soroban da Lógica - teste de validade dual}

Para o teste de validade dual de argumentos utilizar-se-á o reverso das figuras empregadas para o teste de validade. Desse modo, simultaneamente ao teste da validade de argumento com o anverso das figuras, está-se testando a validade dual do argumento dual com o reverso das figuras.

Foi visto, na seção anterior, que o dual de um literal é o próprio literal. Portanto, o reverso de um quadrado é idêntico ao seu anverso.

Por testar-se validade dual com o reverso de figuras, o reverso da barra de inferência é uma barra de inferência dual. Isso será expresso mediante uma barra cujo extremo esquerdo contém um sinal '-‘.

O dual da disjunção inclusiva é a conjunção, portanto a contiguidade de quadrados, formando um bloco, corresponderá à conjunção dos literais correspondentes aos quadrados contíguos.

O dual da conjunção é a disjunção inclusiva, portanto a separação de blocos corresponderá à disjunção inclusiva dos conjuntivos pelos quais estão os blocos separados.

As regras de manipulação das figuras continuam exatamente as mesmas, a despeito da nova interpretação das figuras. Claro, não se trata mais de regras de aferição de informação, mas de regras de aferição de alternativa de informação errada; e a regra de comércio de informação do anverso é, aqui no reverso, interpretada como regra de comércio de alternativa de informação errada.

A Figura 5 apresenta a prova da validade dual de instância de dual de modus ponens cujas premissas, em forma normal disjuntiva, são $\left(\neg \mathrm{p}_{1} \wedge \mathrm{p}_{2}\right)$ e $\mathrm{p}_{1}$, e cuja conclusão é p2. Compare-se com a prova de validade de instância de modus ponens da Figura 2.

$\mathrm{Na}$ próxima seção tratarei dos modos segundo os quais o método pode ser aplicado, dos modos segundo os quais o jogo pode ser praticado. 


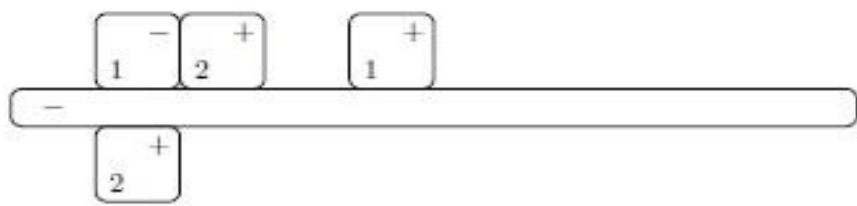

(a)

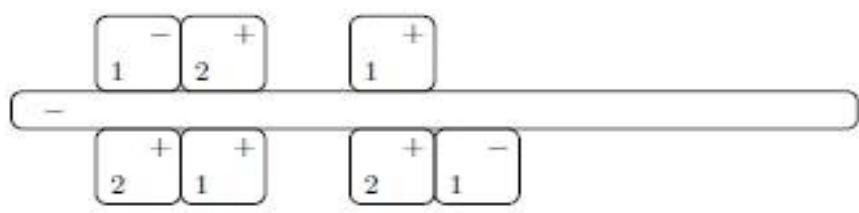

(b)

(c)

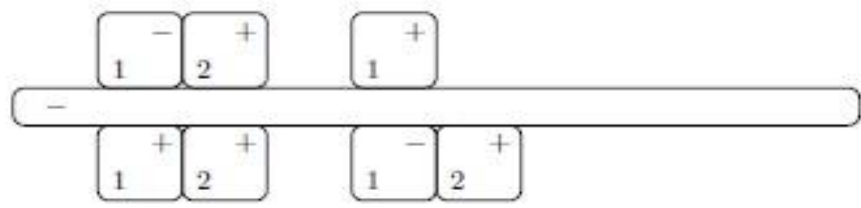

(d)

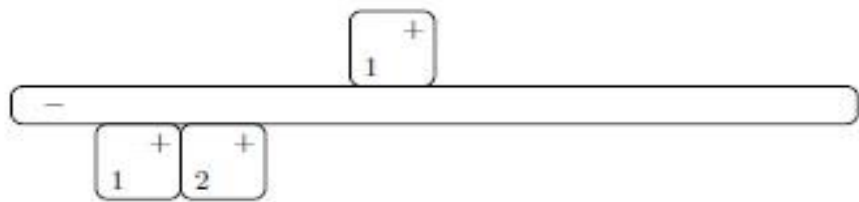

(e)
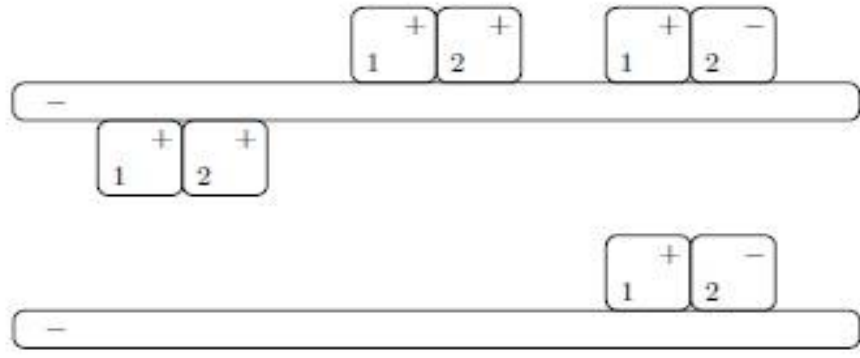

(f)

Figura 5: Passos de prova da validade dual de instância de dual de modus ponens.

\section{Solitário, cooperativo, ou competitivo}

O 'jogo da Lógica' é, geralmente, praticado solitariamente: um único indivíduo compara e manipula premissas e conclusão para determinar a 
validade ou invalidade de argumentos. Há, porém, exceções; por exemplo, a Lógica Dialógica de Paul Lorenzen e Kuno Lorenz (1978) ${ }^{16}$.

Pode-se estabelecer ao menos as seguintes três maneiras distintas de proceder com o método heterogêneo de prova aqui exposto (em qualquer uma dessas maneiras pode-se proceder mediante o exame do anverso ou do reverso):

1. Procedimento solitário: um único usuário do método é responsável pela manipulação tanto de premissas como da conclusão.

2. Procedimento cooperativo: o método é aplicado por dois usuários, um dos quais é responsável pela manipulação das premissas, enquanto que o outro é responsável pela manipulação da conclusão. Os dois usuários não se comunicam entre si e alternam turnos; um turno consiste na aplicação da regra de comércio de informação (regra de comércio de alternativa de informação errada, no caso da validade dual), de uma regra de aferição de informação (regra de aferição de alternativa de informação errada, no caso da validade dual) ou, simplesmente, passar o turno para o outro usuário (quando se entende que qualquer aplicação de regra aos quadrados pelos quais se é responsável não é vantajosa).

3. Procedimento competitivo: o método é aplicado por duas ou mais equipes de usuários, cada qual sendo responsável pelo comércio e aferição de informação (alternativa de informação errada, no caso da validade dual) do mesmo argumento, e cada equipe pode proceder solitária ou cooperativamente.

O procedimento, em qualquer dos casos, dá-se por concluído quando há o esvaziamento da conclusão (o argumento é válido, se utilizar-se o anverso, e é válido dual, se utilizar-se o reverso), ou se chegar a uma configuração em que claramente não se pode esvaziar a conclusão (o argumento é inválido, se utilizar-se o anverso, e é inválido dual, se utilizar-se o reverso).

No caso do procedimento competitivo, há ao menos duas maneiras básicas de medir o desempenho das equipes: ou se utiliza o menor tempo necessário para esvaziar a conclusão ou chegar a uma situação em que claramente não se pode esvaziá-la, ou se utiliza o número mínimo de aplicações de regras necessário para esvaziar a conclusão ou chegar a uma situação em que claramente não se pode esvaziá-la.

Evidentemente, os mesmos procedimentos (solitário, cooperativo, competitivo) e as mesmas medidas de desempenho se aplicam mesmo que o

${ }^{16}$ No Prefácio de seu manual de lógica, Lewis Carroll (1886) aconselha o seguinte em relação à aplicação do seu método heterogêneo de prova: 'At the same time, though one Player is enough, a good deal more amusement may be got by two working at it together, and correcting each other's mistakes.' 
método seja aplicado independente de sua interpretação lógica, ou seja, como um puro jogo.

\section{Hierarquia de níveis de complexidade de argumentos}

That purpose and end [of a system of logical symbols] is simply and solely the investigation of the theory of logic, and not at all the construction of a calculus to aid the drawing of inferences. These two purposes are incompatible, for the reason that the system devised for the investigation of logic should be as analytical as possible, breaking up inferences into the greatest possible number of steps, and exhibiting them under the most general categories possible; [...] (HARTSHORNE; WEISS, 373).

Na citação acima C. S. Peirce pressupõe dois propósitos distintos e aparentemente incompatíveis de investigação de um sistema lógico de símbolos - visando a investigação da teoria lógica ou visando o cálculo, a realização de inferências - e esclarece em que consiste a primeira finalidade e quais as condições se requer sejam satisfeitas para a sua bem sucedida realização. No primeiro caso a finalidade é teórica; no segundo caso, prática. Por finalidade prática se entende dispor de todos os meios para facilitar a realização de inferências, mesmo ao custo da existência de redundâncias e de procedimentos ad hoc.

O método aqui exposto atende a essa finalidade prática; aplicados os devidos pré-processamentos, que não são complicados, a aplicação do método é fácil, a aplicação a uns poucos argumentos, válidos e inválidos, válidos duais e inválidos duais, será o suficiente, para um usuário com o mínimo de destreza, reconhecer essa facilidade. Mas o método também é apto ao propósito de investigação da própria teoria lógica.

O método conta com apenas cinco regras operacionais e uma regra estrutural, e a ordem de aplicação de tais regras na verificação da validade ou invalidade (validade dual ou invalidade dual) de um argumento é, na imensa maioria dos casos, óbvia. Poder-se-ia, com base nelas, fornecer uma medida da complexidade de argumentos válidos (válidos duais) e, a partir dessa medida, estabelecer uma hierarquia de complexidade de argumentos válidos (válidos duais). Minha sugestão é adotar uma medida diretamente proporcional ao número mínimo de regras necessárias para a demonstração da validade (validade dual) e inversamente proporcional ao número de distintos types de sentenças atômicas que o argumento contém. Por exemplo, a instância de modus ponens cujas premissas são $(\neg \mathrm{p} \vee \mathrm{q})$ e p, e cuja conclusão é q, para p e q sentenças atômicas, requer quatro aplicações de regras operacionais e duas aplicações da regra estrutural, totalizando seis aplicações de regras, e envolve duas sentenças atômicas, o que lhe daria três como medida (seis dividido por 
dois); já a instância de eliminação da conjunção cuja única premissa é $(p \wedge q)$ e cuja conclusão é p, requer uma única aplicação da regra estrutural, o que lhe daria meio como medida (um dividido por dois). É claro, poder-se-ia incluir na medida as etapas de pré-processamento do argumento, o que nos exemplos acima não se aplica.

Poder-se-ia objetar que se pode fazer o mesmo com qualquer outro método que opere à base de múltiplas regras. Em relação a isso, contra-objeto que nem todo método que opera à base de múltiplas regras tem a naturalidade do método aqui exposto, pois o método aqui desenvolvido tem uma interpretação natural com base em manipulação de informação (alternativa de informação errada).

Essa hierarquia de argumentos válidos (válidos duais) poderia ser útil, por exemplo, na compreensão dos mecanismos subjacentes ao cometimento de erros lógicos, mas isso é tema para um próximo trabalho.

\section{Referências}

CARROLL, L. The Game of Logic. London: Macmillan, 1886.

CORCORAN, J. "Information-theoretic logic”. In: MARTÍNEZ, C.; RIVAS, U.; VILLEGAS-FORERO (orgs.) Truth in Perspective. Aldershot: Ashgate, p. 113-135, 1998.

FLORIDI, L. "Outline of a Theory of Strongly Semantic Information". Mind and machines, v. 14, n. 2, p. 197-222, 2004.

HARTSHORNE, C.; WeISS, P. Collected Papers of Charles Sanders Peirce.v. 4. Cambridge: Harvard University Press, 1933. A citação dessa obra, utilizada no texto, referir-se-á, como usual, ao parágrafo.

KNEALE, W; KNEALE, M. O desenvolvimento da lógica. $3^{\text {a }}$. edição. Lisboa: Fundação Calouste Gulbenkian, 1991.

LORENZEN, P; LORENZ, K. Dialogische Logik. Darmstadt: Wissensschaftliche Buchgesellschaft, 1978.

SANZ, W. de C. Falsity preservation. CLE e-Prints, v. 8, n. 2, 2008, 14 p. 
AUTOR. A dinâmica da argumentação sob uma perspectiva lógica. Dissertatio, v. 35, p. 195-207, 2012.

AUTOR. "Un tema de Hilbert y Ackermann: Formas normales para laprueba de validez". In: ESQUISABEL, O. M.; AUTOR, F. T. (orgs.) Conocimiento simbólico y conocimiento gráfico. Historia y teoría. Buenos Aires: Academia Nacional de Ciencias de Buenos Aires, p. 71-79, 2013.

AUTOR; SANZ, W. C. Teorias axiomáticas: o problema da ampliação da base. Fundamento: revista de filosofia, v. 1, p. 51-61, 2013.

AUTOR; MENDONÇA, B. R. Argumentos exuberantes e sua retificação. Analytica, v. 18, p. 109-121, 2014.

AUTOR. Herbrand e a silogística ampliada. Philósophos, v. 20, p. 125-144, 2015.

Email: ftsautter@ufsm.br

Recebido: 08/2016

Aprovado: 09/2016 